INVESTIGACIÓN

Recibido: 19/04/2018 --- Aceptado: 29/04/2019 --- Publicado: 15/09/2019

\title{
LA INDUSTRIA DEL JUGUETE EN FACEBOOK. EL ENGAGEMENT CON LOS USUARIOS DURANTE LA CAMPAÑA DE NAVIDAD 2014-15
}

\section{The toy industry at Facebook. The engagement with users during the 2014-15 Christmas campaign}

(D) $8 \mathbb{R}^{6}$ Jesús Díaz-Campoํ․ Universidad Internacional de la Rioja (UNIR). España. jesus.diaz@unir.net

(D) (3) $\mathbb{R}^{2}$ Erika Fernández-Gómez². Universidad Internacional de la Rioja (UNIR). España. erika.fernandez@unir.net

\section{RESUMEN}

Esta investigación presenta un análisis de contenido realizado sobre los mensajes que publicaron las marcas más importantes de la industria del juguete en España de acuerdo al Programa Estrella de la Asociación Española de Fabricantes de Juguetes (AEFJ) en la red social Facebook durante la campaña de Navidad 2014-2015. En concreto, se analizaron los mensajes publicados por Famosa, Playmobil, Comansi, Hasbro, Barbie y Fisher-Price. El principal objetivo de la investigación ha sido analizar la interacción entre las marcas y sus seguidores. Se tiene en cuenta la finalidad de las publicaciones y el empleo de recursos multimedia (vídeos, fotografías, etiquetas, emoticonos o enlaces a páginas web). En total se recogieron 991 mensajes. Los resultados desglosan las características que presentan los diez mensajes más populares (con más likes), los diez que registran más participación (es decir, lo que recibieron un mayor número de comentarios) y los diez más virales (los que se compartieron más veces). La estrategia empleada por este sector se basa en transmitir mensajes de carácter informativo o saludar a la audiencia a través de mensajes que son acompañados de una fotografía.

\footnotetext{
${ }^{1}$ Jesús Díaz-Campo: Licenciado y doctor en Periodismo por la Universidad Complutense de Madrid. Director del Máster en Comunicación e Identidad Corporativa de la Universidad Internacional de La Rioja (UNIR). Acreditado a profesor titular por ANECA.

2 Erika Fernández-Gómez: Doctora en Comunicación por la Universidad de Vigo. Profesora adjunta en la Universidad Internacional de la Rioja (UNIR), donde imparte docencia en el área de Empresa y Comunicación. Miembro del grupo de investigación COYSODI de UNIR.
} 
Díaz-Campo, J. y Fernández-Gómez, E. La industria del juguete en Facebook. El engagement con los usuarios durante la campaña de Navidad 2014-15

Estos mensajes se corresponden con los que más gustan y se comparten. Por el contrario, los contenidos que más se comentan son aquellos que buscan la interacción con los usuarios a través de mecanismos como los concursos.

PALABRAS CLAVE: Facebook - juguetes - engagement - comunicación corporativa redes sociales - campaña de Navidad - marca.

\section{ABSTRACT}

This piece of research presents a content analysis carried out on the messages published by the most important brands of the toy industry in Spain according to the Estrella Program of the Spanish Association of Toy Manufacturers (AEFJ) on the Facebook social network during the 2014-2015 Christmas campaign. Specifically, the messages published by Famosa, Playmobil, Comansi, Hasbro, Barbie and Fisher-Price were analyzed. The main objective of this piece of research has been to analyze the interaction between brands and their followers. The purpose of the publications and the use of multimedia resources (videos, photographs, labels, emoticons or links to web pages) are taken into account. In total, 991 messages were collected. The results break down the characteristics of the ten most popular messages (with the most I like it), the ten with more participation (that is, the ones that received the largest number of comments) and the ten most viral (those that were shared more times). The strategy employed by this sector is based on transmitting informative messages or greeting the audience through messages that are accompanied by a photograph. These messages correspond to those that their users like and share most. On the contrary, the contents that are most commented are those that seek interaction with users through mechanisms such as contests.

KEY WORDS: Facebook - toys - engagement - corporative communication - social media - Christmas campaign - brand.

\section{A INDÚSTRIA DOS BRINQUEDOS NO FACEBOOK. O ENGAGEMENT COM OS USUÁRIOS DURANTE A CAMPANHA DE NATAL DE 2014/2015}

\section{RESUME}

Esta investigação apresenta uma analises de conteúdo realizado sobre as mensagens que publicaram as marcas mais importantes da indústria de brinquedos na Espanha de acordo ao programa "Estrella de la Asociacion Española de Fabricantes de Juguetes (AEFJ) na rede social Facebook durante a campanha de Natal de 2014/2015. Em concreto, se analisaram as mensagens publicadas por Famosa, Playmobil, Comansi, Hasbro, Barbie e Fisher-Prince. O principal objetivo desta investigação foi analisar a interação entre as marcas e seus seguidores. Foi levado em conta a finalidade das publicações e o emprego de recursos multimídia (vídeos, fotografias, etiquetas, emojis

Vivat Academia. Revista de Comunicación. 15 septiembre 2019 /15 diciembre 2019, nº 148, 1-21 

con los usuarios durante la campaña de Navidad 2014-15

ou enlaces às páginas web). Em total se reuniram 991 mensagens. Os resultados separam as características que apresentam as dez mensagens mais populares, com mais likes, os dez que registram mais participação (isto e, o que receberam um maior número de comentários) e os dez, mas virais (os que foram mais compartidos). A estratégia empregada por este setor se baseia em transmitir mensagens de caráter informativo ou brindar audiência através de mensagens que são acompanhadas de uma fotografia. Estas mensagens correspondem-se com os que mais gostam e se compartem. Pelo contrário, os conteúdos que mais se comentam são aqueles que buscam a interação com os usuários através de mecanismos como os concursos.

PALAVRAS CHAVE: Facebook - brinquedos - engagement - comunicação corporativa redes sociais - campanha de Natal - marca.

\section{Como citar el artículo:}

Díaz-Campo, J. y Fernández-Gómez, E. (2019). La industria del juguete en Facebook. El engagement con los usuarios durante la campaña de Navidad 2014-15. [The toy industry at Facebook. The engagement with users during the 2014-15 Christmas campaign]. Vivat Academia. Revista de Comunicación, 148, 1-21.

DOI: http://doi.org/10.15178/va.2019.148.1-21

Recuperado de http://www.vivatacademia.net/index.php/vivat/article/view/1120

\section{INTRODUCCIÓN}

Un $85 \%$ de los internautas españoles cuyas edades se sitúan entre 16 y 65 años utilizan las redes sociales. El 87\% de ellos utilizan Facebook a diario y para ello emplean una media de una hora diaria. Por otro lado, ocho de cada diez usuarios siguen a alguna marca y el 39\% de ellos lo hace con bastante frecuencia. Asimismo, un 57\% de usuarios confiesa haber sido influido por las redes sociales cuando realizan sus compras. (IAB Spain y Elogia, 2018).

A la luz de estos datos, parece claro que el medio digital en general y las redes sociales en particular se han convertido en un escenario idóneo para las marcas a la hora de promocionarse y poder interactuar con sus seguidores. $Y$ es que en los últimos años se ha pasado de un sistema basado en los medios, en el que no existe posibilidad de respuesta, a un proceso de comunicación fundamentado en el diálogo (Martínez Estremera, 2012, p. 9).

Del mismo modo, los usuarios en su faceta de consumidores dedican una parte significativa de su tiempo a desarrollar en las redes sociales acciones relacionadas de una forma $\mathrm{u}$ otra con el consumo, en un proceso en el cual no les importa compartir su estilo de visita y sus preferencias de consumo con aquellos otros usuarios con los que están en consumo online (Ashley y Tuten, 2015; Dimitriu y Guesalaga, 2015).

Vivat Academia. Revista de Comunicación. 15 septiembre 2019 /15 diciembre 2019, nº 148, 1-21 

con los usuarios durante la campaña de Navidad 2014-15

Entre esos usuarios con los que están en contacto están las propias marcas, que de este modo tienen la oportunidad de relacionarse con sus potenciales consumidores de una forma nunca antes vista, y por ello a menudo no dudan en establecer con ellos conversaciones en la red (Sashittal, Hodis y Sriramachandramurthy, 2014).

Uno de los aspectos que deben tener en cuenta las marcas a la hora de diseñar esa estrategia es el hecho de que los usuarios pueden llegar a estar saturados de información debido a la cantidad de mensajes que reciben a diario de los medios de comunicación, tanto los tradicionales como los digitales (Sashittal, Hodis y Sriramachandramurthy, 2014).

A la hora precisamente de tratar de entender el comportamiento de los usuarios en las redes sociales, se han realizado diversas clasificaciones de perfiles (entre otros, Malhotra, Malhotra y See, 2013; Campbell, Ferraro y Sands, 2014; Min-Sook; Jong-Kuk y Yong, 2015). De entre todas ellas, para los propósitos de la presente investigación resulta de especial utilidad la llevada a cabo por Hodis, Sriramachandramurthy y Sashittal (2015), ya que se refiere de manera concreta a Facebook. Estos autores identifican cuatro grandes grupos atendiendo al comportamiento general en la red social y, en especial a la creación de contenido (actualización del estado, publicación de imágenes y comentarios, etc.) $\mathrm{y}$, por otro lado, al consumo de contenidos (navegar por los distintos posts, dar "me gusta", compartir o comentar esos posts, etc.). Los cuatro grupos son los siguientes:

- Devotos (crean y consumen mucho contenido)

- Buscadores de conexión (consumen mucho pero crean poco contenido)

- Buscadores de atención (consumen poco pero crean mucho contenido)

- Cazadores de entretenimiento (consumen y crean poco contenido)

Como puede verse, la mayoría de los usuarios son activos de un modo u otro. Pues bien, esa actividad y ese diálogo que ya se ha mencionado propicia que se dé un paso más y que se produzca el compromiso (engagement) por parte del usuario hacia la marca (Dimitriu y Guesalaga, 2017). Un compromiso que puede explicarse por la teoría del apego, es decir, la necesidad del ser humano de mantener vínculos afectivos permanentes con otros seres humanos que, en el caso de las redes sociales, se extiende también a las marcas, en tanto en cuanto éstas interactúan con los usuarios (Hart, Nailling, Bizer, y Collins, 2015).

El sector del juguete no es una excepción a esta tendencia. Si bien los catálogos siguen siendo uno de los principales medios utilizados por los padres cuando estos pretenden buscar información sobre los productos que piensan adquirir para sus hijos, un $64 \%$ de ellos reconoce ya recurrir al medio digital para obtener esa información, mientras que casi una cuarta parte $(23 \%)$ los adquiere a través de algún medio online. Del mismo modo, el 31\% de los padres admite que consulta los comentarios de otros usuarios antes de dar el paso final y comprar y un $39 \%$ se fija especialmente en las fotografías de los

Vivat Academia. Revista de Comunicación. 15 septiembre 2019 /15 diciembre 2019, n 148, 1-21 
Díaz-Campo, J. y Fernández-Gómez, E. La industria del juguete en Facebook. El engagement con los usuarios durante la campaña de Navidad 2014-15

juguetes que puede consultar en la red (Asociación Española de Fabricantes de Juguetes, The Cocktayl Analysis e IAB Spain, 2012).

Este protagonismo de Internet y las redes sociales se acrecienta cuando se hace referencia al sector del juguete y se sitúa el ámbito de estudio en la campaña de Navidad. Teniendo en cuenta la estacionalidad de ventas del sector y la concentración publicitaria durante la campaña navideña (Rom y Sabaté, 2009, p. 73), muchas marcas para las que los menores constituyen uno de sus segmentos de mercado fundamentales (Bringué, 2001) centran buena parte de sus esfuerzos en diseñar la mejor estrategia posible para esa campaña.

Asimismo no debemos olvidar que en este sector una de las particularidades de la comunicación publicitaria de las marcas radica en que el niño es el usuario del producto, si bien, el menor debe influir en la decisión de compra y por ello en muchas ocasiones el público al que realmente se dirige la campaña son los padres (Tur y López Sánchez, 2012). Las empresas también deben convencer a los padres en las redes sociales, pues a pesar de que muchos menores cuenten con su propio perfil, la edad mínima para abrir una cuenta en Facebook es 14 años, con lo que hablaríamos ya de un público adolescente frente a uno infantil, con edades entre 4 y 12 años.

De hecho, buena parte de las investigaciones que han estudiado la publicidad para menores se centran en analizar las campañas navideñas, tanto en España como en el ámbito internacional (entre otros, Bradbard, 1985; Bradbard y Parkman, 1985; Consejo Audiovisual de Andalucía e Instituto Andaluz de la Mujer, 2013; Díaz-Campo y Fernández-Gómez, 2017; Fernández, 2004; Ferrer, 2007; Gil y Pérez, 2012; Granizo López, 2017; Martínez y Nicolás, 2015; Martínez y Nicolás, 2016; Martínez, Nicolás y Gaona, 2014; Martínez, Nicolás y Salas, 2013; Martínez Pastor, Pérez-Ugena y Salas, 2011; Martínez Pastor, Gaona y Nicolás, 2017; Martínez Pastor y Salas, 2012; Moncada Lara, 2013; Observatorio Andaluz de la Publicidad no sexista, 2014; Pérez-Ugena, Martínez y Salas, 2011; Pine y Nash, 2001; Pine, Wilson y Nash, 2007, Rubio, 2011).

La mayoría de ellos adopta una perspectiva ética-deontológica y se centra en analizar si existe algún tipo de estereotipos de género en los anuncios. Asimismo, el estudio de hábitos que promueve en los niños este tipo de publicidad o la presencia de familiares adultos en esos anuncios, y las relaciones con se establecen entre ellos y los menores (Martínez Pastor, Nicolás y García Manso, 2016) son otras de las cuestiones que han tratado de abordar estos trabajos.

No obstante, se trata de estudios que se centran en soportes como, principalmente, la televisión y, en segundo término, los catálogos de juguetes. Por el contrario, el medio digital y las redes sociales no han sido estudiados con tanto detalle, y mucho menos

Vivat Academia. Revista de Comunicación. 15 septiembre 2019 /15 diciembre 2019, nº 148, 1-21 

con los usuarios durante la campaña de Navidad 2014-15

desde la perspectiva del engagement, a pesar de que éste se considera como uno de los principales objetivos que las marcas deberían buscan en las redes sociales.

Así, Solanas y Carreras (2011, p. 80) explican que este concepto hace referencia a cómo en un panorama en el que se produce una saturación de mensajes, el objetivo no sólo debe ser conseguir impactar al consumidor, sino que además, hay que intentar dar un paso más y lograr que éste interactúe con la marca. Por tanto, el engagement habla del grado de compromiso en las conversaciones. Y las principales métricas de la interacción de usuarios en Facebook son el botón "me gusta", la opción compartir o comentar (Huertas, Setó-Pámies y Míguez-González, 2014, p. 17).

Estas mismas métricas son señaladas por IAB Spain (2012) en el Libro Blanco de la Comunicación en Medios Sociales, al considerar que el impacto de la actividad de las marcas en medios sociales se puede medir a través de variables cuantitativas. Así, el número de fans y la interacción con los contenidos de la página son un indicativo de cómo una empresa consigue implicar a sus seguidores (IAB Spain, 2012, p. 25).

En ese sentido, los pocos estudios realizados hasta el momento con el objetivo de analizar la estrategia de las marcas de juguetes en las redes sociales (Fernández-Goméz y Díaz-Campo, 2015; Uchoa-Craveiro, 2012) o aquellos otros que incluyen ese sector entre los que conforman el objeto de estudio (entre otros, Furlow, 2011; Gatautis, Vitkauskaitė y de Reuver, 2017; Hassan y Casaló Ariño, 2016; Hoffman, Novak y Kang, 2017) apuntan a una mayor interacción con los seguidores, más allá del objetivo inicial de proporcionar información de carácter comercial a los seguidores.

\section{OBJETIVOS}

Esta investigación tiene como objetivo principal analizar las características de los mensajes más populares, más virales y que han logrado una mayor participación de entre todos los publicados por las marcas de juguetes en Facebook. De este modo, se pretende determinar cómo caracterizan las marcas sus mensajes para generar engagement con sus seguidores. Para alcanzar este objetivo, se plantean las siguientes hipótesis:

H1. Las marcas que cuentan con un mayor número de seguidores en Facebook consiguen una mayor engagement en sus mensajes. Contar con más seguidores en el perfil supone que los mensajes publicados lleguen a un público más numeroso, algo que a su vez podría traducirse también en un mayor engagement de esos mensajes.

H2. Los mensajes más populares, con mayor participación y que más se comparten son los que invitan a los seguidores a participar en concursos promovidos por las marcas. Los mensajes que las marcas publican en sus perfiles persiguen diversos

Vivat Academia. Revista de Comunicación. 15 septiembre 2019 /15 diciembre 2019, nº 148, 1-21 
objetivos, como promocionar el producto, buscar la interacción o promover un concurso en el que participen los seguidores, entre otros. A priori, esta última es una de las opciones más atractivas para el público que sigue a las marcas en Facebook.

H3. El uso de recursos multimedia en los mensajes, tales como fotografías o vídeos, contribuye a generar un mayor engagement con los seguidores. Las redes sociales posibilitan que las marcas empleen un mayor número de recursos que en otros soportes tradicionales como la televisión o la prensa escrita. Junto a las fotografías o los vídeos, los mensajes pueden incluir enlaces a una página web o hashtags, que pueden aumentar el interés por el contenido que se publica.

\section{METODOLOGÍA}

Para poder verificar o falsar las hipótesis anteriores, se realizó un análisis de contenido de los mensajes que publicaron los fabricantes de juguetes a lo largo la campaña navideña 2014-2015. Para seleccionar la muestra de marcas analizadas, se siguió un triple criterio:

a) Formar parte de la Asociación Española de Fabricantes de Juguetes (AEFJ), cuyos socios suponen el $87 \%$ de facturación del sector juguetero en España.

b) Haber sido incluido en el Programa Estrella de AEFJ, que en Navidad selecciona los juguetes que considera que representan una mayor novedad y destacan más.

c) Contar con un perfil en Facebook.

De este modo, en un primer momento, se eligieron las ocho marcas a las que pertenecían los juguetes seleccionados para el Programa Estrella de AEFJ: Bizak, Comansi, Famosa, Giochi-Preziosi, Hasbro, IMC Toys, Mattel y Playmobil.

De esta primera selección se descartaron las marcas Bizak, IMC Toys y GiochiPreziosi porque en el momento de realizar la investigación no tenían perfil en Facebook. Mientras, en el caso de Mattel, que tampoco tiene perfil en esta red social, se consideró que está formada por marcas que sí están presentes en Facebook y que, además, cuentan con productos en el Programa Estrella de AEFJ, como Barbie y Fisher-Price.

Por tanto, la muestra definitiva quedó conformada por:

- Barbie. Marca que se basa en una muñeca que fabrica la empresa Mattel.

- Comansi. Marca española especializada en juguetes que se dirigen a bebés y menores con edades comprendidas entre 0 y 8 años.

- Famosa. Marca española que comenzó fabricando muñecos y que después ha diversificado su producción, que ahora incluye figuras, juegos de mesa, etc.

- Fisher-Price. Marca que fabrica juguetes para bebés.

- Hasbro. Marca especializada en juegos de mesa.

- Playmobil. Marca basada en una figura con una altura de 7,5 centímetros.

Vivat Academia. Revista de Comunicación. 15 septiembre 2019 /15 diciembre 2019, n 148, 1-21 

con los usuarios durante la campaña de Navidad 2014-15

Se realizó un análisis de contenido de los mensajes publicados en estas páginas entre el 1 de noviembre de 2014 y el 31 de enero de 2015, es decir, durante la campaña promocional de Navidad. Hay que señalar que se contabilizaron únicamente los mensajes publicados por las propias marcas, pero no los comentarios que realizaron otros usuarios.

Tabla 1. Muestra de páginas analizadas.

\begin{tabular}{|l|l|}
\hline Barbie & https://www.facebook.com/officialbarbieespana \\
\hline Comansi & https://es-es.facebook.com/juguetescomansi \\
\hline Famosa & https://www.facebook.com/famosajuguetes \\
\hline Fisher-Price & https://www.facebook.com/FisherPriceEspana \\
\hline Hasbro Gaming & https://www.facebook.com/HasbroGamingES \\
\hline Playmobil & https://es-es.facebook.com/playmobilespana \\
\hline
\end{tabular}

Fuente: Elaboración propia.

Los mensajes fueron caracterizados de acuerdo a dos variables:

- El objetivo del mensaje. En esta variable se diferenciaron un total de cuatro categorías: mensaje informativo o saludo a los usuarios que visitan la página de la marca (por ejemplo, felicitando la Navidad o el Año Nuevo o realizándoles algún tipo de recomendación o consejo); promoción de un juguete; juego o concurso (por ejemplo, sorteo de un pack de juguetes entre quienes acierten una pregunta relacionada con la marca) o mensaje publicado para fomentar la participación (por ejemplo "Haz clic en me gusta si...").

- Respecto a los recursos empleados, se recogió la información relativa a si los mensajes publicados por las marcas incluían, vídeos, fotografías, etiquetas (hashtags), emoticonos o enlaces a páginas web. En este último caso se diferenció si se trataba de un enlace que dirigía a la propia página de la marca o bien llevaba al usuario a una web externa. Por lo que respecta a los vídeos, se contabilizaron todos aquellos que se podían poner en marcha directamente desde la página de Facebook de la marca, pero no se tuvieron en cuenta aquellos que requerían enlazar a otra página para poder ser vistos y que, por lo tanto, no ofrecían la posibilidad de contabilizar el número de reproducciones.

Estas dos variables fueron empleadas en un estudio previo para determinar el engagement de las cadenas de televisión infantil y juvenil en España (Fernández-Gómez y Díaz-Campo, 2014).

Como se ha mencionado antes, el objetivo del presente estudio es determinar las características de los mensajes más populares, que han logrado una mayor participación en los perfiles estudiados y aquellos que más se han compartido. Por ello, también se cuantificaron estos datos en cada uno de los mensajes analizados: la popularidad

Vivat Academia. Revista de Comunicación. 15 septiembre 2019 /15 diciembre 2019, n 148 , 1-21 
Díaz-Campo, J. y Fernández-Gómez, E. La industria del juguete en Facebook. El engagement con los usuarios durante la campaña de Navidad 2014-15

(número de "me gusta" recibidos), la participación (número de comentarios generados) y la viralidad (número de veces que el mensaje fue compartido).

\section{RESULTADOS}

En total se recogieron 991 mensajes. Los resultados que se exponen a continuación desglosan las características que presentan los diez mensajes más populares, los diez que registran más participación y los diez más virales. Estos datos nos llevan a determinar qué estrategia emplea este sector para lograr el engagement de su audiencia en las redes sociales.

Tabla 2. Número total de mensajes publicados y seguidores por marca.

\begin{tabular}{|c|c|c|}
\hline Marca & Mensajes & Seguidores \\
\hline Famosa & 510 & 81.583 \\
\hline Fisher-Price & 120 & 2.924 .094 \\
\hline Hasbro Gaming & 118 & 310.876 \\
\hline Comansi & 99 & 6.871 \\
\hline Playmobil & 96 & 120.355 \\
\hline Barbie & 48 & 13.071 .884 \\
\hline Total & 991 & 16.515 .643 \\
\hline
\end{tabular}

Fuente: Elaboración propia.

En la tabla 2 se aprecia como la actividad de las marcas en Facebook no se corresponde con el tener un amplio número de seguidores en este perfil. Así, la marca con mayor número de entradas registradas en el período analizado corresponde a Famosa. Le sigue con una actividad muy inferior Fisher-Price y en tercer lugar, Hasbro Gaming. Si bien, es la marca con menor número de publicaciones la que tiene una mayor comunidad de seguidores: Barbie. Sí existe relación en el caso de los juguetes para bebés ya que, Fisher-Price es la segunda empresa en número de seguidores y la segunda en número de posts publicados.

Al analizar el objetivo perseguido por los mensajes publicados por las marcas de juguetes durante la campaña de Navidad se ha encontrado que, el 54,9\% de los mensajes corresponden a contenidos de carácter informativo o que persiguen saludar a los usuarios (por ejemplo, para felicitarles la Navidad). En segundo lugar, pero con un porcentaje menor, el 21,8\% de los mensajes persiguen la promoción de productos. Por su parte, los juegos y concursos representan el 13,5\% y los mensajes que buscan estimular la participación son los menos utilizados por los anunciantes en sus páginas con un $9,7 \%$.

En el caso de los recursos empleados por las marcas, el 26\% de las marcas han empleado la fotografía como recursos principal de sus publicaciones. El emoticono representa el

Vivat Academia. Revista de Comunicación. 15 septiembre 2019 /15 diciembre 2019, n 148, 1-21 
segundo recurso, presente en el $24 \%$ de los mensajes. El hashtag está presente en el $23 \%$ de las ocasiones y el enlace a la web propia en el $20 \%$. La web externa fue empleada en el $5 \%$ de las ocasiones y el video en un $2 \%$.

Si comparamos estos datos con el objetivo perseguido por los 10 mensajes más populares (tabla 3), es decir, los que consiguen un mayor número de "me gusta", vemos como también 5 de estas publicaciones tienen una finalidad informativa. La promoción está presente con dos mensajes al igual que el estimular la participación. Si bien, el concurso solo está presente una vez. La marca dirigida a bebés es la que presenta un mayor número de publicaciones que gustan a los fans. Precisamente a esta marca corresponde el concurso con el sorteo de la casa de Minnie donde se invita a los seguidores a demostrar lo que saben sobre este personaje de Disney.

Tabla 3. Objetivo y recursos empleados en los 10 mensajes más populares.

\begin{tabular}{|c|c|c|c|c|}
\hline Marca & Mensaje & $\begin{array}{c}\text { Me } \\
\text { gusta }\end{array}$ & Objetivo & Recursos \\
\hline $\begin{array}{l}\text { Fisher- } \\
\text { Price }\end{array}$ & $\begin{array}{l}\text { Ellos tienen la capacidad de sorprendernos... desde el } \\
\text { primer momento. }\end{array}$ & 14536 & $\begin{array}{r}\text { Informar- } \\
\text { saludar }\end{array}$ & Foto \\
\hline $\begin{array}{l}\text { Fisher- } \\
\text { Price }\end{array}$ & $\begin{array}{l}\text { No te pierdas el especial de Mickey y Minnie en Disney } \\
\text { Channel España con motivo de su cumpleaños. Una } \\
\text { programación muy especial para homenajear a estos } \\
\text { queridísimos personajes. Este domingo a partir de las } \\
\text { 7:00h. ¡Feliz cumpleaños Mickey y Minnie! }\end{array}$ & 13662 & $\begin{array}{r}\text { Informar- } \\
\text { saludar }\end{array}$ & $\begin{array}{r}\text { Foto } \\
\text { Enlace web } \\
\text { externa }\end{array}$ \\
\hline $\begin{array}{l}\text { Fisher- } \\
\text { Price }\end{array}$ & $\begin{array}{l}\text { Tú ya tienes el mejor regalo para esta navidad. } \\
\text { ¡Disfrútalo! }\end{array}$ & 13286 & $\begin{array}{r}\text { Informar- } \\
\text { saludar }\end{array}$ & $\begin{array}{r}\text { Foto } \\
\text { Emoticono }\end{array}$ \\
\hline $\begin{array}{l}\text { Fisher- } \\
\text { Price }\end{array}$ & $\begin{array}{l}\text { Año nuevo, nuevos nacimientos. Padres recién } \\
\text { estrenados de todo el mundo comparten con nosotros } \\
\text { los primeros deseos para sus bebés }\end{array}$ & 8986 & $\begin{array}{r}\text { Informar- } \\
\text { saludar }\end{array}$ & $\begin{array}{r}\text { Foto } \\
\text { Vídeo } \\
\text { Emoticono }\end{array}$ \\
\hline $\begin{array}{l}\text { Fisher- } \\
\text { Price }\end{array}$ & $\begin{array}{l}\text { ¿Quieres ganar la casa de Minnie de Fisher-Price? Entra } \\
\text { aquí http:// fpfami.ly/0AmLR y demuestra que lo } \\
\text { sabes todo sobre Minnie. }\end{array}$ & 7719 & Concurso & $\begin{array}{r}\text { Foto } \\
\text { Enlace web } \\
\text { propia }\end{array}$ \\
\hline $\begin{array}{l}\text { Fisher- } \\
\text { Price }\end{array}$ & $\begin{array}{l}\text { Mientras sostienes este regalo en tus brazos solo tienes } \\
\text { un deseo. Compártelo con nosotros. } \\
\text { \#DeseoParaMiBebé }\end{array}$ & 5360 & Participación & Foto \\
\hline $\begin{array}{l}\text { Hasbro } \\
\text { Gaming }\end{array}$ & $\begin{array}{l}\text { Los juegos de mesa son geniales para disfrutar del } \\
\text { tiempo con la familia o los amigos. }\end{array}$ & 5011 & Promoción & $\begin{array}{r}\text { Foto } \\
\text { Enlace web } \\
\text { propia }\end{array}$ \\
\hline $\begin{array}{l}\text { Fisher- } \\
\text { Price }\end{array}$ & $\begin{array}{l}\text { ¡Empieza la cuenta atrás! } \\
\text { Hoy nos comemos la primera chocolatina del } \\
\text { calendario de adviento }\end{array}$ & 4627 & $\begin{array}{r}\text { Informar- } \\
\text { saludar }\end{array}$ & Foto \\
\hline Barbie & $\begin{array}{l}\text { ¡Por fin es Nochevieja! Es el momento de despedir el } \\
2014 \text { con una gran fiesta y empezar el nuevo año. ¿Qué } \\
\text { buenos propósitos tienes para el 2015? }\end{array}$ & 4454 & Participación & Foto \\
\hline $\begin{array}{l}\text { Fisher- } \\
\text { Price }\end{array}$ & $\begin{array}{l}\text { El top de nuestra carta a Papá Noel - ¡Gimnasio piano } \\
\text { pataditas! Con sus pies podrá tocar el piano dando } \\
\text { pataditas y con sus manitas podrá jugar con los }\end{array}$ & 4426 & Promoción & $\begin{array}{r}\text { Foto } \\
\text { Enlace web } \\
\text { propia } \\
\end{array}$ \\
\hline
\end{tabular}


Díaz-Campo, J. y Fernández-Gómez, E. La industria del juguete en Facebook. El engagement con los usuarios durante la campaña de Navidad 2014-15

divertidos móviles que cuelgan. ¿Tu peque está listo

para marcar el ritmo? http://fpfami.ly/0Db65

Fuente: Elaboración propia.

Por otro lado, atendiendo a los recursos empleados, se puede ver como todos los mensajes que encabezan el ranking de los más populares se caracterizan por publicarse junto a una fotografía. El vídeo aparece en una sola ocasión, al igual que el enlace a la web externa. El redirigir a los usuarios a la página web está presente en tres ocasiones y el emoticono en dos.

Observando los mensajes que más comentarios han suscitado (tabla 4) se observa que solo uno de los mensajes más populares (tabla 3) ha sido al mismo tiempo uno de los más comentados. Es el caso de la publicación de Hasbro con la promoción de que los juegos de mesa son una buena forma de disfrutar con la familia y los amigos. Como se puede ver, ha recibido un total de 1,607 comentarios.

Tabla 4. Objetivo y recursos de los 10 mensajes más comentados.

\begin{tabular}{|c|c|c|c|c|}
\hline Marca & Mensaje & Comentarios & Objetivo & Recursos \\
\hline $\begin{array}{l}\text { Hasbro } \\
\text { Gaming }\end{array}$ & $\begin{array}{l}\text { Los juegos de mesa son geniales para disfrutar del } \\
\text { tiempo con la familia o los amigos. }\end{array}$ & 1607 & Promoción & $\begin{array}{r}\text { Foto } \\
\text { Enlace web } \\
\text { propia }\end{array}$ \\
\hline Famosa & $\begin{array}{l}\text { ¡Moteros y moteras! Jugad con nosotros y } \\
\text { decidnos cómo termina esta frase tan molona } \\
\text { Emoticono smile \#FamoAdivina } \\
\text { Aquí va una pequeña pista: } \\
\text { http:/ / bit.ly / 1wiUCJn }\end{array}$ & 1095 & Concurso & $\begin{array}{r}\text { Foto } \\
\text { Enlace web } \\
\text { propia } \\
\text { Hashtag } \\
\text { Emoticono } \\
\end{array}$ \\
\hline Famosa & $\begin{array}{l}\text { ¡Estrenamos nuestro calendario de Adviento! } \\
\text { \#FamoAdivina } \\
\text { Si adivinas cómo termina la frase escrita en esta } \\
\text { foto "Mete masas en el horno...", podrás } \\
\text { convertirte en el primer ganador del mes. } \\
\text { PISTA (En uno de estos } 2 \text { enlaces encontrarás la } \\
\text { respuesta): } \\
\text { http:// bit.ly/1pFRI09 } \\
\text { http:// bit.ly/1vak0Ri }\end{array}$ & 1044 & Concurso & $\begin{array}{r}\text { Foto } \\
\text { Enlace web } \\
\text { propia } \\
\text { Hashtag }\end{array}$ \\
\hline $\begin{array}{l}\text { Hasbro } \\
\text { Gaming }\end{array}$ & $\begin{array}{l}\text { ¡Hoy es el ÚLTIMO DÍA de nuestro CONCURSO! } \\
\text { Coméntanos qué juego deseas para estas } \\
\text { Navidades y podrás conseguirlo. Si estás indecis@ } \\
\text { sobre cuál elegir te dejamos nuestro catálogo: } \\
\text { http:/ / goo.gl/0fyRbd ¿Sabías que... este año } \\
\text { Monopoly celebra su } 80 \text { cumpleaños? Pero } 80 \text { no } \\
\text { es nada, si los comparas con los millones de } \\
\text { partidas jugadas y las miles de horas de diversión } \\
\text { en todo el mundo. \#Feliz80AniversarioMonopoly }\end{array}$ & 984 & Concurso & $\begin{array}{r}\text { Foto } \\
\text { Enlace web } \\
\text { propia }\end{array}$ \\
\hline Famosa & ¡Nuevo acertijo que solo los mejores doctores & 955 & Concurso & Foto \\
\hline
\end{tabular}

Vivat Academia. Revista de Comunicación. 15 septiembre 2019 /15 diciembre 2019, nº 148, 1-21 
Díaz-Campo, J. y Fernández-Gómez, E. La industria del juguete en Facebook. El engagement con los usuarios durante la campaña de Navidad 2014-15

\begin{tabular}{|c|c|c|c|c|}
\hline & $\begin{array}{l}\text { sabrán responder! } \\
\text { \#FamoAdivina cómo termina esta frase } \\
\text { Emoticono smile } \\
\text { A)Dormidito } \\
\text { B)Tranquilito } \\
\text { C)Tapadito } \\
\text { Pista: http:/ / bit.ly / } 1 \mathrm{vNq} 4 \mathrm{y} 0\end{array}$ & & & $\begin{array}{r}\text { Enlace web } \\
\text { propia } \\
\text { Hashtag } \\
\text { Emoticono }\end{array}$ \\
\hline $\begin{array}{l}\text { Hasbro } \\
\text { Gaming }\end{array}$ & $\begin{array}{l}\text { Supera este Reto de “Ciencia y Naturaleza” y } \\
\text { podrás ganar un Trivial Edición Familia } \\
\text { PREGUNTA: ¿Los caballos duermen de pie. } \\
\text { ¿Verdadero o falso? Danos tu respuesta antes del } \\
\text { próximo MARTES } 2 \text { a las 23:59H. }\end{array}$ & 906 & Concurso & Foto \\
\hline Famosa & $\begin{array}{l}\text { ¿Quién no tiene hambre a estas horas? } \\
\text { \#FamoAdivina cómo termina esta frase y podrás } \\
\text { ir al restaurante de Barriguitas a disfrutar de su } \\
\text { menú. Emoticono smile } \\
\text { Aquí encontrarás una pista: } \\
\text { http:/ / bit.ly/1xaBFe5 }\end{array}$ & 897 & Concurso & $\begin{array}{r}\text { Foto } \\
\text { Enlace web } \\
\text { propia } \\
\text { Hashtag } \\
\text { Emoticono }\end{array}$ \\
\hline Famosa & $\begin{array}{l}\text { Viajando se descubren lugares excepcionales y se } \\
\text { viven momentos muy especiales Emoticono smile } \\
\text { \#FamoAdivina cómo termina esta frase de Nancy } \\
\text { la aventurera. } \\
\text { Una pequeña pista por aquí: } \\
\text { http:/ / bit.ly/1wKynw7 }\end{array}$ & 884 & Concurso & $\begin{array}{r}\text { Foto } \\
\text { Enlace web } \\
\text { propia } \\
\text { Hashtag } \\
\text { Emoticono }\end{array}$ \\
\hline $\begin{array}{l}\text { Hasbro } \\
\text { Gaming }\end{array}$ & $\begin{array}{l}\text { Supera este Reto de "Deporte y Ocio" y podrás } \\
\text { ganar un Trivial Edición Familia PREGUNTA: } \\
\text { ¿De cuántas casillas o escaques se compone un } \\
\text { tablero de ajedrez? Danos tu respuesta antes del } \\
\text { próximo MARTES } 16 \text { a las 23:59H. }\end{array}$ & 825 & Concurso & Foto \\
\hline Famosa & $\begin{array}{l}\text { ¡El día tan esperado ha llegado! Ya hemos } \\
\text { cumplido el Calendario de Adviento y queremos } \\
\text { acabar con un último acertijo de \#FamoAdivina } \\
\text { de lo más viajero Emoticono smile } \\
\text { ¿Sabrías decirnos cómo termina esta rima? } \\
\text { Aquí encontrarás la respuesta: } \\
\text { http:/ / bit.ly/1vmYchO }\end{array}$ & 825 & Concurso & $\begin{array}{r}\text { Foto } \\
\text { Enlace web } \\
\text { propia } \\
\text { Hashtag } \\
\text { Emoticono }\end{array}$ \\
\hline
\end{tabular}

Fuente: Elaboración propia.

En este caso es Famosa la marca que más comentarios recibe a las publicaciones que ha realizado. Aunque cabe mencionar que Hasbro Gaming también ha conseguido una alta participación por parte de sus seguidores. Destaca entre otros aspectos el hecho de que todos los mensajes más comentados tienen como objetivo el promover un juego o concurso salvo en el primer caso mencionado, que por el contrario se basa en la promoción. Para ello se emplean un mayor número de recursos ya que, aunque la fotografía sigue siendo la que está presente en todas las publicaciones, destaca que existe también un enlace a la web de la marca. De este modo se comprueba que para

Vivat Academia. Revista de Comunicación. 15 septiembre 2019 /15 diciembre 2019, nº 148, 1-21 
Díaz-Campo, J. y Fernández-Gómez, E. La industria del juguete en Facebook. El engagement con los usuarios durante la campaña de Navidad 2014-15

poder concursar o jugar es necesario visitar la página principal del fabricante de juguetes. Aparece en esta ocasión el hashtag y destaca asimismo la ausencia del vídeo.

Tabla 5. Objetivo y recursos de los 10 mensajes más virales.

\begin{tabular}{|c|c|c|c|c|}
\hline Marca & Mensaje & Compartido & Objetivo & Recursos \\
\hline $\begin{array}{l}\text { Fisher- } \\
\text { Price }\end{array}$ & $\begin{array}{l}\text { Año nuevo, nuevos nacimientos. Padres recién } \\
\text { estrenados de todo el mundo comparten con } \\
\text { nosotros los primeros deseos para sus bebés }\end{array}$ & 1677 & $\begin{array}{r}\text { Informar- } \\
\text { saludar }\end{array}$ & $\begin{array}{r}\text { Foto } \\
\text { Vídeo } \\
\text { Emoticono } \\
\end{array}$ \\
\hline $\begin{array}{l}\text { Fisher- } \\
\text { Price }\end{array}$ & $\begin{array}{l}\text { No te pierdas el especial de Mickey y Minnie en } \\
\text { Disney Channel España con motivo de su } \\
\text { cumpleaños. Una programación muy especial para } \\
\text { homenajear a estos queridísimos personajes. Este } \\
\text { domingo a partir de las 7:00h. ¡Feliz cumpleaños } \\
\text { Mickey y Minnie! }\end{array}$ & 1256 & $\begin{array}{l}\text { Informar- } \\
\text { saludar }\end{array}$ & $\begin{array}{r}\text { Foto } \\
\text { Enlace web } \\
\text { externa }\end{array}$ \\
\hline $\begin{array}{l}\text { Hasbro } \\
\text { Gaming }\end{array}$ & $\begin{array}{l}\text { Los juegos de mesa son geniales para disfrutar del } \\
\text { tiempo con la familia o los amigos. }\end{array}$ & 709 & Promoción & $\begin{array}{r}\text { Foto } \\
\text { Enlace web } \\
\text { propia }\end{array}$ \\
\hline $\begin{array}{l}\text { Fisher- } \\
\text { Price }\end{array}$ & $\begin{array}{l}\text { Ellos tienen la capacidad de sorprendernos... } \\
\text { desde el primer momento. }\end{array}$ & 700 & $\begin{array}{r}\text { Informar- } \\
\text { saludar }\end{array}$ & Foto \\
\hline $\begin{array}{l}\text { Fisher- } \\
\text { Price }\end{array}$ & $\begin{array}{l}\text { Tú ya tienes el mejor regalo para esta Navidad. } \\
\text { ¡Disfrútalo! }\end{array}$ & 567 & $\begin{array}{r}\text { Informar- } \\
\text { saludar }\end{array}$ & $\begin{array}{r}\text { Foto } \\
\text { Emoticono }\end{array}$ \\
\hline $\begin{array}{l}\text { Fisher- } \\
\text { Price }\end{array}$ & $\begin{array}{l}\text { ¿Quieres ganar la casa de Minnie de Fisher-Price? } \\
\text { Entra aquí http://fpfami.ly/0AmLR y demuestra } \\
\text { que lo sabes todo sobre Minnie. }\end{array}$ & 476 & Concurso & $\begin{array}{r}\text { Foto } \\
\text { Enlace web } \\
\text { propia }\end{array}$ \\
\hline $\begin{array}{l}\text { Fisher- } \\
\text { Price }\end{array}$ & $\begin{array}{l}\text { ¡Empieza la cuenta atrás! } \\
\text { Hoy nos comemos la primera chocolatina del } \\
\text { calendario de adviento }\end{array}$ & 394 & $\begin{array}{l}\text { Informar- } \\
\text { saludar }\end{array}$ & Foto \\
\hline Famosa & $\begin{array}{l}\text { ¡Estrenamos nuestro calendario de Adviento! } \\
\text { \#FamoAdivina } \\
\text { Si adivinas cómo termina la frase escrita en esta } \\
\text { foto "Mete masas en el horno...", podrás convertirte } \\
\text { en el primer ganador del mes. } \\
\text { PISTA (En uno de estos } 2 \text { enlaces encontrarás la } \\
\text { respuesta): } \\
\text { http:/ / bit.ly/1pFRI09 } \\
\text { http:// bit.ly/1vak0Ri }\end{array}$ & 378 & Concurso & $\begin{array}{r}\text { Foto } \\
\text { Enlace web } \\
\text { propia } \\
\text { Hashtag }\end{array}$ \\
\hline $\begin{array}{l}\text { Hasbro } \\
\text { Gaming }\end{array}$ & $\begin{array}{l}\text { Con Monopoly Empire puedes ser el dueño de } \\
\text { todo un imperio al alcance de tus dados...Pídelo } \\
\text { como deseo en nuestro concurso y comparte } \\
\text { tiempo con amigos estas navidades. jSortearemos } \\
12 \text { juegos de mesa! Descubre los juegos más } \\
\text { deseados y elige el tuyo en http://goo.gl/0fyRbd }\end{array}$ & 353 & Concurso & Foto \\
\hline Comansi & $\begin{array}{l}\text { Buenas tardes, empezamos un nuevo \#concurso } \\
\text { donde sorteamos un \#pack completo de figuritas } \\
\text { de Peppa Pig incluyendo las \#novedades que } \\
\text { podéis ver en este post. Para participar tenéis que } \\
\text { compartir este post y responder a la pregunta: } \\
\text { ¿Qué es lo que más os gusta de Peppa Pig? }\end{array}$ & 350 & Concurso & $\begin{array}{r}\text { Foto } \\
\text { Enlace web } \\
\text { externa } \\
\text { Hashtag }\end{array}$ \\
\hline
\end{tabular}


Díaz-Campo, J. y Fernández-Gómez, E. La industria del juguete en Facebook. El engagement con los usuarios durante la campaña de Navidad 2014-15

\begin{tabular}{|l|l|l|l|l|}
\hline & $\begin{array}{l}\text { Haremos el sorteo el próximo miércoles día 28 } \\
\text { ¡Suerte! }\end{array}$ & & & \\
\hline
\end{tabular}

Fuente: Elaboración propia.

Por último, siete de los mensajes más populares (véase tabla 3) son también los más compartidos (tabla 5). De modo que, Fisher-Price también lidera el número de mensajes viralizados. Si bien, en esta ocasión el objetivo que se persigue es en primer lugar informar o saludar y en segundo promover un juego o concurso. La promoción solo está presente en una ocasión. La fotografía sigue siendo el recurso presente en todos los mensajes que se comparten. Se emplean enlaces a la web propia, a la web externa, emoticonos y hashtags, siendo el vídeo el recurso menos empleado al estar presente en un solo uno de los mensajes más virales.

\section{DISCUSIÓN}

Los datos obtenidos en el estudio nos permiten extraer las siguientes conclusiones con respecto a cada una de las tres hipótesis formuladas en la primera parte de esta investigación:

Las marcas con más seguidores consiguen mejor engagement. Tras el análisis de los mensajes más populares, comentados y compartidos se aprecia que no es la marca con más seguidores (Barbie) la que mayor engagement consigue de la audiencia. Esta marca solo está presente entre los diez mensajes con más "me gusta" con un mensaje en el que pregunta a los usuarios cuáles son sus propósitos para el año nuevo. Si bien, la tercera marca en número de seguidores es la que consigue más likes y que sus contenidos se compartan.

Los comentarios están presentes en las marcas que promueven juegos o concursos, como es el caso de Famosa y Hasbro Gaming que representan la segunda y cuarta marca en seguidores. De modo que, aunque es cierto que las marcas que tienen más seguidores consiguen mejores datos de participación por parte de sus públicos que las que tienen menos, no hay una relación directa de cuantos más fans más engagement.

Lo que sí se evidencia es que no es suficiente con estar presente en las redes sociales y contar con un amplio número de seguidores. Barbie es la marca más seguida pero no cuenta con una estrategia de comunicación en la misma, al ser la que menos mensajes ha publicado. Esto tiene como consecuencia la falta de engagement con sus seguidores.

Facebook es un medio bidireccional, y por ello aquellas marcas que buscan la interacción con sus seguidores a través de juegos y concursos son las que consiguen una mejor implicación de los mismos.

Vivat Academia. Revista de Comunicación. 15 septiembre 2019 /15 diciembre 2019, n 148 , 1-21 

con los usuarios durante la campaña de Navidad 2014-15

Los mensajes más populares, comentados y compartidos son los que se basan en concursos. Se aprecia que los mensajes más comentados sí son los concursos, en segundo lugar los concursos consiguen un buen grado de viralidad. Si bien, tienen una menor presencia en el caso de la popularidad. A los seguidores les gusta y comparten aquellos contenidos de carácter informativo o que están dirigidos a saludarles. El carácter promocional genera poca participación por parte de los fans de las marcas de juguetes.

Pero se aprecia que los concursos son una buena forma de conseguir una comunidad activa en esta red social. Así, se valora la información y el promover la participación frente a los mensajes puramente comerciales. Lo que refuerza la idea de que la presencia en las redes sociales debe basarse en un diálogo y en ofrecer algo más a los usuarios.

Los mensajes que recurren a la fotografía y al vídeo consiguen un mejor engagement. Solo se puede afirmar en el caso de la imagen, que está presente en todos los mensajes más populares, comentados y compartidos. Sin embargo, el video es poco empleado por las marcas. Las compañías jugueteras aprovechan su presencia en las redes sociales para redirigir el tráfico a sus páginas web y consiguen buenos resultados de participación de sus seguidores especialmente cuando se trata de concursos. Lo que pone de manifiesto la existencia de una estrategia en la que la página web de las marcas sigue siendo un elemento importante para la comunicación de las marcas. Si bien este tráfico se consigue estando presente en redes sociales.

Así, tras el análisis de las publicaciones realizadas por las marcas de juguetes en Facebook a lo largo de la campaña de Navidad 2014-2015 se puede determinar que la estrategia de estas compañías en la red social está bastante alineada con las motivaciones de los usuarios de redes sociales. Al igual que señala el informe de IAB Spain y Elogia (2017), los contenidos que más gustan y se comparten son informativos, respondiendo así a la motivación de seguir a las marcas para mantenerse informados. Al mismo tiempo, las publicaciones más comentadas y también más virales responden a la segunda razón para seguir a una marca, el participar en un concurso.

Además, si bien el carácter promocional no es el que mayor engagement consigue de la audiencia, teniendo en cuenta que los padres valoran las fotografías de los juguetes antes de comprar, la imagen es un recurso presente en todos los mensajes más populares, comentados y compartidos. En ese sentido, la red social es una buena oportunidad para que las marcas se comuniquen con sus públicos pero no por ello se deja de lado el website pues, en la estrategia de las marcas de juguetes se observa el redirigir el tráfico hacia las páginas web.

Ha cambiado la forma en la que el consumidor quiere relacionarse con las marcas, y en este estudio se observa como muchas de las marcas estudiadas del sector juguete ha sabido adaptarse al nuevo medio.

Vivat Academia. Revista de Comunicación. 15 septiembre 2019 /15 diciembre 2019, nº 148, 1-21 
Díaz-Campo, J. y Fernández-Gómez, E. La industria del juguete en Facebook. El engagement con los usuarios durante la campaña de Navidad 2014-15

Por último, este estudio podría ser replicado en campañas navideñas de años posteriores, de forma similar a como sucede con los anuncios en televisión, para observar la posible evolución de las tendencias detectadas y, al mismo tiempo, abre la puerta a la realización en el futuro de otros similares que tengan también Facebook como objeto de estudio pero se refieran a diferentes sectores o bien al sector juguetes en redes incipientes como es Instagram Otra forma interesante de abordar el engagement es a través de encuestas o focus group a los seguidores de estos perfiles, con el objetivo de conocer los mecanismos que activan su implicación con estas marcas.

\section{REFERENCIAS}

Ashley, C. y Tuten, T. (2015). Creative strategies in social media marketing: An exploratory study of branded social content and consumer engagement. Psychology $\mathcal{E}$ Marketing, 32, 15-27.

Recuperado de http://www.academia.edu/download/42154764/Research_Work__Creative_Strategies_in_Social_Media_Marketing.pdf

Asociación Española de Fabricantes de Juguetes; The Cocktayl Analysis y IAB Spain (2012). Estudio sobre niños, juguetes e Internet.

Recuperado de http://tcanalysis.com/blog/posts/estudio-sobre-ninos-juguetes-einternet.

Bradbard, M. (1985). Sex Differences in Adults' gifts and Children's Toy Requests at Christmas. Psychological Reports, 56, 691-696.

DOI: http://doi.10.2466/pr0.1985.56.3.969

Bradbard, M. y Parkman, S. A. (1985). Gender Differences in Preschool Children's Toy Requests. Journal of Genetic Psychology, 145, 283-284.

DOI: http://doi.10.1080/00221325.1984.10532277

Bringué, X. (2001). Publicidad infantil y estrategia persuasiva: un análisis de contenido, Zer. Revista de Estudios de Comunicación, 6(10), 107-129. Recuperado de http://www.ehu.eus/ojs/index.php/Zer/article/view/6104

Campbell, C.; Ferraro, C., y Sands, S. (2014). Segmenting consumer reactions to social network marketing. European Journal of Marketing, 48, 432-452.

Consejo Audiovisual de Andalucía e Instituto Andaluz de la Mujer (2013). Los canales temáticos concentran más del $90 \%$ de los anuncios de juguetes en la campaña de Navidad. Recuperado de https://bit.ly/2qKhnch.

Vivat Academia. Revista de Comunicación. 15 septiembre 2019 /15 diciembre 2019, nº 148, 1-21 
Díaz-Campo, J. y Fernández-Gómez, E. La industria del juguete en Facebook. El engagement con los usuarios durante la campaña de Navidad 2014-15

Díaz-Campo, J. y Fernández-Gómez, E. (2017). Estereotipos de género y socialización del menor: La publicidad de juguetes a través de Facebook en España. Observatorio $\left(O B S^{*}\right), 11(3)$, 25lpage-43.

Recuperado de http://obs.obercom.pt/index.php/obs/article/view/923

Dimitriu, R., y Guesalaga, R. (2017). Consumers' social media brand behaviors: uncovering underlying motivators and deriving meaningful consumer segments. Psychology \& Marketing, 34(5), 580-592.

Fernández, C. (2004). Navidad y publicidad de juguetes: La autorregulación del sector. Estrategias de Comunicación y Marketing, 125, 74.

Fernández-Gómez, E. y Díaz-Campo, J. (2014). Los canales temáticos infantiles y juveniles en Facebook: análisis de los perfiles de Disney Channel, Boing y Neox. Comunicación y hombre, 10, 179-194. Recuperado de http://www.comunicacionyhombre.com/pdfs/10_inve_fernandezydiaz.pdf

Fernández-Gómez, E. y Díaz- Campo, J. (2015). La estrategia de marketing del sector del juguete en Facebook: Análisis de la campaña de navidad 2014 en España. Prisma Social: revista de investigación social, 14, 124-151. Recuperado de http://www.redalyc.org/html/3537/353744530005/

Ferrer, M. (2007). Los anuncios de juguetes en la campaña de Navidad. Comunicar, 15(29), 135-142. Recuperado de http://www.redalyc.org/html/158/15802923/

Furlow, N. E. (2011). Find us on Facebook: how cause marketing has embraced social media. Journal of Marketing Development and Competitiveness, 5(6), 61.

Gatautis, R.; Vitkauskaitė, E. y de Reuver, M. (2017). Use of Facebook and Google Platforms for SMEs Business Model Innovation. BLED 2017 Proceedings. 38.

Gil, L. y Pérez, E. (2012). Publicidad, estereotipos y roles de juego desde una perspectiva de género. Análisis de catálogos de juguetes de El Corte Inglés 2010-2011 (Trabajo Fin de Máster). Universidad Complutense de Madrid. Recuperado de eprints.ucm.es/16605

Granizo López, M. (2017). Publicidad e infancia. Análisis narrativo de los anuncios de los juguetes más vendidos en 2015 (Trabajo de Fin de Grado). Universidad de Granada. Facultad de Ciencias de la Educación. Recuperado de http:// citeseerx.ist.psu.edu/viewdoc/download?doi=10.1.1.728.3028\&rep=rep1\&typ e=pdfhttp://digibug.ugr.es/handle/10481/46269\#.Wk3nuLiYFSQ

Vivat Academia. Revista de Comunicación. 15 septiembre 2019 /15 diciembre 2019, nº 148, 1-21 
Díaz-Campo, J. y Fernández-Gómez, E. La industria del juguete en Facebook. El engagement con los usuarios durante la campaña de Navidad 2014-15

Hart, J., Nailling, E., Bizer, G. Y., y Collins, C. K. (2015). Attachment theory as a framework for explaining engagement with Facebook. Personality and Individual Differences, 77, 33-40. Recuperado de http:// citeseerx.ist.psu.edu/viewdoc/download?doi=10.1.1.728.3028\&rep=rep1\&typ $\mathrm{e}=\mathrm{pdf}$

Hassan, M. y Casaló Ariño, L. V. (2016). Consumer devotion to a different height: How consumers are defending the brand within Facebook brand communities. Internet Research, 26(4), 963-981.

Hodis, M. A., Sriramachandramurthy, R., y Sashittal, H. C. (2015). Interact with me on my terms: A four segment Facebook engagement framework for marketers. Journal of Marketing Management, 31(11-12), 1255-1284.

Hoffman, D. L.; Novak, T. P. y Kang, H. (2017). Let's Get Closer: Feelings of Connectedness from Using Social Media, with Implications for Brand Outcomes. Journal of the Association for Consumer Research, 2(2), 216-228.

Huertas, A.; Setó-Pàmies, D. y Míguez-González, M. I. (2015). Comunicación de destinos turísticos a través de los medios sociales. El profesional de la información, 24(1). DOI: 10.3145/epi.v24i1.33135

IAB Spain (2012). Libro blanco de la comunicación en medios sociales. Recuperado de https://bit.ly/2vw4ibu

IAB Spain y Elogia (2018). Estudio Anual de Redes Sociales 2018. Recuperado de https:/ /iabspain.es/wp-content/uploads/estudio-redes-sociales-2018_vreducida.pdf

Malhotra, A.; Malhotra, C. K. y See, A. (2013). How to create brand engagement on Facebook. MIT Sloan Management Review, 54, 18-20.

Martínez, E. y Nicolás, M. Á. (2015). Informe de la publicidad de juguetes en TV durante las navidades de 2014-15. Análisis de los spots a la luz de los códigos deontológicos y jurídicos. Madrid: OMM Campus Libros.

Martínez, E. y Nicolás, M. Á. (2016). Publicidad y juguetes: Un análisis de la aplicación de los códigos deontológicos y jurídicos en los spots de juguetes durante la navidad 2015-2016. Madrid: OMM Campus Libros.

Martínez, E.; Nicolás, M. Á. y Salas, Á. (2013). La representación de género en las campañas de publicidad de juguetes en Navidades (2009-12). Comunicar, 21(41), 187194. Recuperado de http:/ /www.redalyc.org/html/158/15828675020/

Vivat Academia. Revista de Comunicación. 15 septiembre 2019 /15 diciembre 2019, n 148 , 1-21 
Díaz-Campo, J. y Fernández-Gómez, E. La industria del juguete en Facebook. El engagement con los usuarios durante la campaña de Navidad 2014-15

Martínez Estremera, J. I. (2012). Introducción a los medios sociales. Community Manager: gestión de comunidades virtuales, 8-16. Recuperado de http://www.aercomunidad.org/publicaciones/gestion-de-comunidades-virtuales/

Martínez Pastor, E., Gaona, C., y Nicolás, M. Á. (2017). Gestión pública y privada de la publicidad de juguetes. Regulación y autorregulación en la Unión Europea y España. Gestión y política pública, 26(2), 453-490.

Martínez Pastor, E.; Nicolás Ojeda, M. A. y Gaona, C. (2014). Informe de la publicidad de juguetes en televisión dirigidas a los menores durante las navidades 2013-2014: Desde el punto de vista deontológico y jurídico. Recuperado de http:/ / www.aefj.es/ noticias/?id=394

Martínez Pastor, E.; Nicolás Ojeda, M. A. y García Manso, A. (2016). A theoretical review of the child socialisation process in Spain. Prisma Social: revista de investigación social, 1, 378-399.

Martínez Pastor, E. Pérez-Ugena, Á. y Salas, Á. (2011). Publicidad y juguetes: Análisis de los códigos deontológicos y jurídicos. Pensar la Publicidad. Revista Internacional de Investigaciones Publicitarias, 4(2), 127-140.

Martínez Pastor, E. y Salas, A. (2012). Informe sobre la publicidad de juguetes de la campaña navideña emitida en televisión durante las navidades del 2012. Recuperado de http:/ / www.publiteca.es/2012/04/informe-de-la-publicidad-de-juguetes.html

Min-Sook, P., Jong-Kuk, S., \& Yong, J. (2015). A taxonomy of social networking site users: Social surveillance and self surveillance perspective. Psychology $\mathcal{E}$ Marketing, $32,601-610$

Moncada Lara, D. M. (2013) ¿La televisión es el factor determinante para la decisión de compra de los niños entre los 5 y 8 años en los estratos 2 y 3 de la ciudad de Bogotá? (Tesis doctoral). Facultad de Ciencias Económicas. Especialidad en Mercadeo de Servicios. Universidad Militar Nueva Granada. Recuperado de http:// repository.unimilitar.edu.co/handle/10654/7098

Observatorio Andaluz de la Publicidad no sexista (2014). Informe sobre la Campaña de Juegos y Juguetes 2014. Recuperado de https://bit.ly/2HA58bO

Pérez-Ugena y Coromina, Á.; Martínez Pastor, E. y Salas Martínez, A. (2011). Los estereotipos de género en la publicidad de los juguetes. Ámbitos, 20, 217-235.

Vivat Academia. Revista de Comunicación. 15 septiembre 2019 /15 diciembre 2019, n 148, 1-21 
Díaz-Campo, J. y Fernández-Gómez, E. La industria del juguete en Facebook. El engagement con los usuarios durante la campaña de Navidad 2014-15

Pine, K. J. y Nash, A. (2002). Dear Santa: The effects of television advertising on young children. International Journal of Behavioral Development. 26(6), 529-539. Recuperado de http://journals.sagepub.com/doi/abs/10.1080/01650250143000481

Pine, K. J.; Wilson, P. y Nash, A. S. (2007). The relationship between television advertising, children's viewing and their requests to Father Christmas. Journal of Developmental and Behavioral Pediatrics, 28, 456-461.

Rom, J. y Sabaté, J. (2009). Con los juguetes no se juega. Quaderns del CAC, 22, 71-79.

Rubio, A. (2011). La publicidad infantil en navidad: ¿fomentan la actividad física y relaciones sociales? Revista Española de Comunicación en Salud, 2(2), 69-77. Recuperado de https:/ / e-revistas.uc3m.es/index.php/RECS/article/view/3394r

Sashittal, H. C., Hodis, M. y Sriramachandramurthy, R. (2014). Is your brand a living entity? MIT Sloan Management Review, 55, 96-95. Recuperado de https://www.researchgate.net/profile/Rajendran_Sriramachandramurthy/publicati on/261098971_Is_Your_Brand_a_Living_Entity/links/53e54d7b0cf2fb748716ba77/IsYour-Brand-a-Living-Entity.pdf

Tur Viñes, V. y López Sánchez, C. (2012). Estrategias comunicativas para los nuevos perfiles sociales de la infancia. Actas del III Congreso Internacional de la Asociación Española de Investigación de la Comunicación (AE-IC), 18-20 de enero de 2012, Tarragona, España. Recuperado de https:// bit.ly/2vqdVsk

Solanas, O. y Carreras, G. (2011). Nuevos medios publicitarios: la influencia del mundo digital en la planificación de medios. Trípodos, 28. 63.81. Recuperado de http://www.raco.cat/index.php/tripodos/article/view/247484

Uchoa-Craveiro, P. S. (2012). Valores diseminados por la muñeca Barbie en la página web barbie.com y en Facebook. Vivat Academia. Revista de Comunicación, 117, $267-290$. Recuperado de http://vivatacademia.net/index.php/vivat/article/view/61

\section{AUTORES}

\section{Jesús Díaz-Campo}

Profesor titular en la Universidad Internacional de la Rioja (UNIR) desde 2011 donde imparte docencia en el área de Comunicación y dirige el Máster Universitario en Comunicación e Identidad Corporativa. Acreditado por ANECA como profesor titular, cuenta con un sexenio de investigación (CNEAI). Es miembro del grupo de investigación Comunicación y Sociedad Digital (COYSODI) de la UNIR y sus principales 
Díaz-Campo, J. y Fernández-Gómez, E. La industria del juguete en Facebook. El engagement con los usuarios durante la campaña de Navidad 2014-15

líneas de investigación son ética y deontología de la comunicación, comunicación corporativa, redes sociales y comunicación de la salud.

jesus.diaz@unir.net

Orcid ID: https:// orcid.org/0000-0001-5014-8749

Google Scholar: https://scholar.google.es/citations?user=rNxx5WYAAAAJ\&hl=es

ResearchGate: https://www.researchgate.net/profile/Jesus_Diaz-campo

Scopus: https://www.scopus.com/authid/detail.uri?authorId=55948255700

Academia.edu:

http://universidadinternacionaldelarioja.academia.edu/Jes\%C3\%BAsD\%C3\%ADazdel

CampoLozano

\section{Erika Fernández-Gómez}

Profesora adjunta en la Universidad Internacional de la Rioja (UNIR) desde 2010 donde imparte docencia en el área de Comunicación. Acreditada por la ANECA como profesora contratada doctora, cuenta con un sexenio de investigación (CNEAI). Es miembro del grupo de investigación Comunicación y Sociedad Digital (COYSODI) de la UNIR y sus principales líneas de investigación son televisión, publicidad, redes sociales, comunicación de salud y audiencia infantil y juvenil.

erika.fernandez@unir.net

Orcid ID: https:// orcid.org/0000-0002-7088-1814

Google Scholar: https://scholar.google.es/citations?user=w3utJpgAAAAJ\&hl=es

ResearchGate: https://www.researchgate.net/profile/Erika_Gomez4

Scopus: https://www.scopus.com/authid/detail.uri?authorId=56041622700

Academia.edu:

http://universidadinternacionaldelarioja.academia.edu/ErikaFern\%C3\%A1ndezG\%C3 $\underline{\% B 3 m e z}$

Vivat Academia. Revista de Comunicación. 15 septiembre 2019 /15 diciembre 2019, nº 148, 1-21 